$$
\text { CONF-961202--27 }
$$

To be submitted to the 1996 Fall MRS meeting

\title{
Linear and Nonlinear Optical Properties of Metal Nanocluster-Silica \\ Composites Formed by Implantation of Sb in High Purity Silica
}

\author{
R. H. Magruder, III, R. A. Weeks, T. S. Anderson \\ Vanderbilt University \\ Nashville, TN \\ R. A. Zuhr \\ Oak Ridge National Laboratory \\ Oak Ridge, TN
}

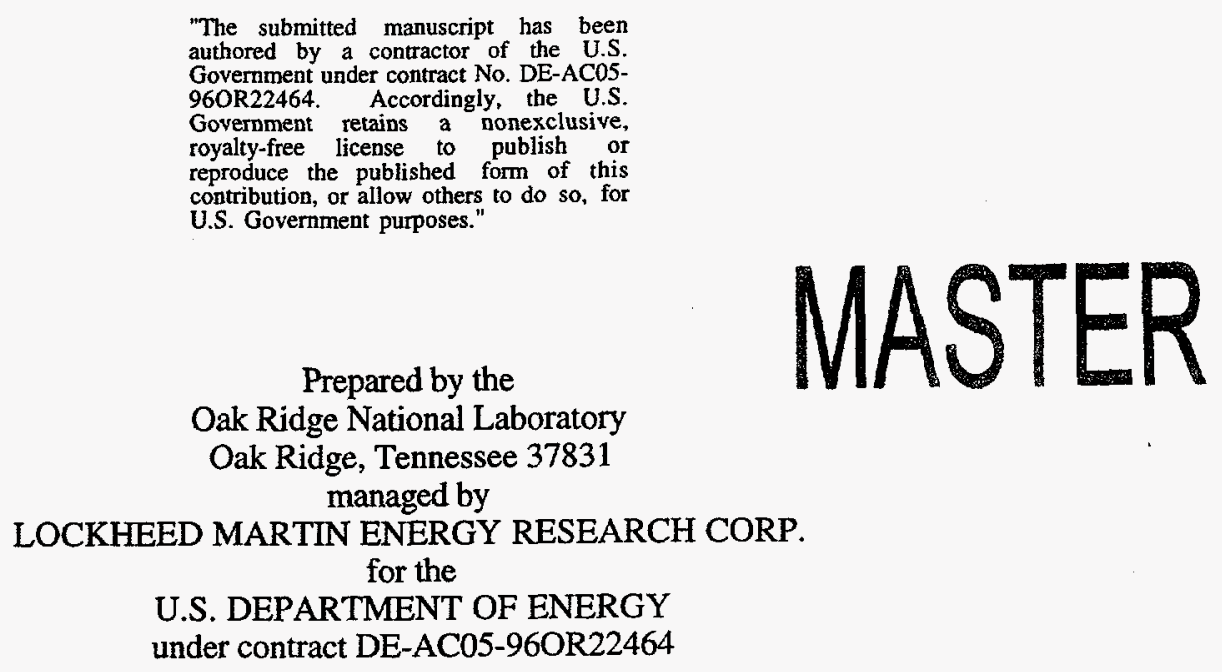

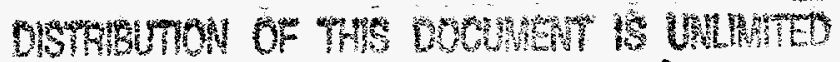

November 1996

DISCLAIMER

This report was prepared as an account of work sponsored by an agency of the United States Government. Neither the United States Government nor any agency thereof, nor any of their employees, makes any warranty, express or implied, or assumes any legal liability or responsibility for the accuracy, completeness, or usefulness of any information, apparatus, product, or process disclosed, or represents that its use would not infringe privately owned rights. Reference herein to any specific commercial product, process, or service by trade name, trademark, manufacturer, or otherwise does not necessarily constitute or imply its endorsement, recommendation, or favoring by the United States Government or any agency thereof. The views and opinions of authors expressed herein do not necessarily state or reflect those of the United States Government or any agency thereof. 


\section{DISCLAMIER}

Portions of this document may be illegible in electronic image products. Images are produced from the best avallable original document. 



\section{DISCLAIMER}

This report was prepared as an account of work sponsored by an agency of the United States Government. Neither the United States Government nor any agency thereof, nor any of their employees, make any warranty, express or implied, or assumes any legal liability or responsibility for the accuracy, completeness, or usefulness of any information, apparatus, product, or process disclosed, or represents that its use would not iniringe privately owned rights. Reference herein to any specific commercial product, process, or service by trade name, trademark, manufacturer, or otherwise does not necessarily constitute or imply its endorsement, recommendation, or favoring by the United States Government or any agency thereof. The views and opinions of authors expressed herein do not necessar. ily state or reflect those of the United States Government or any agency thereof. 

LINEAR AND NONLINEAR OPTICAL PROPERTIES OF METAL NANOCLUSTER-SILICA COMPOSITES FORMED BY IMPLANTATION OF SB IN HIGH PURITY SILICA

R. H. Magruder,III*, R.A. Weeks*, T.S. Anderson* and R. A. Zuhr**

*Dept. of Applied and Engineering Science Vanderbilt University, Nashville, TN

**Solid State Division, Oak Ridge National Laboratory, Oak Ridge, TN.,

\section{ABSTRACT}

The linear and nonlinear optical properties of a series of Sb nanometer dimension metal colloids in silica were measured. The colloids were fabricated by the implantation of Sb in silica with nominal doses of 3,6 and $9 \times 10^{16}$ ions $/ \mathrm{cm}^{2}$ at $320 \mathrm{keV}$. The linear optical response was measured from 200 to $900 \mathrm{~nm}$. The nonlinear optical properties were measured using the $\mathrm{z}$-scan technique at wavelengths of 596 and $617 \mathrm{~nm}$. The results are compared to linear and nonlinear properties of a sample implanted with $6 \times 10^{16} \mathrm{Ag}$ ions $/ \mathrm{cm}^{2}$. Both absorption and the nonlinear index of refraction increase with increasing volume fraction for the $\mathrm{Sb}$ implanted samples. The lower linear absorption and comparable nonlinear index of refraction for the Sb colloid-glass composites may be better for devices than composites using metal colloids that have a surface plasmon resonance.

\section{INTRODUCTION}

Effective medium theory for small non interacting particles in a dielectric $[1,2]$ predicts substantial enhancements of the nonlinear response of the composite for optical frequencies near the surface plasmon resonance (SPR) frequency. This enhancement is due to local field effects and results from dielectric confinement. A significant problem with metal colloid-glass composites for device applications is that both the linear and nonlinear absorption in general increase near the surface plasmon resonance frequency. This increase in absorption has deleterious effects on the relative figures of merit for devices operating near these SPR frequencies using these materials. [3, 4] Even away from the SPR frequencies, linear and nonlinear absorption can limit device possibilities. The ability to form metal colloid-glass composites with large nonlinear responses while exhibiting smaller absorption losses could significantly improve the feasibility of these materials for optical devices.

Here we report the modification of the optical response of nanometer dimension metal colloids formed by the implantation of $\mathrm{Sb}$. Sb was chosen because its electronic structure does not give rise to a surface plasmon resonance in the 200 to $900 \mathrm{~nm}$ wavelength region. $[5,6] \mathrm{We}$ compare the results for the $\mathrm{Sb}$ metal colloids to those for $\mathrm{Ag}$ colloids.

\section{EXPERIMENTAL}

Sb ions were sequentially implanted in Coming 7940 high purity silica substrates. The $\mathrm{Sb}$ ions were implanted at $320 \mathrm{keV}$ with a substrate temperature of $\sim 270^{\circ} \mathrm{K}$ and a current density of $\sim$ $2 \mu$ amps $/ \mathrm{cm}^{2}$. Nominal doses of 3,6 and $9 \times 10^{16}$ ions $/ \mathrm{cm}^{2}$ were used. For comparison an $\mathrm{Ag}$ implanted sample with a nominal dose of $6 \times 10^{16}$ ions $/ \mathrm{cm}^{2}$ was fabricated. The Ag ions were implanted at $305 \mathrm{keV}$ with substrate temperatures of $\sim 270^{\circ} \mathrm{K}$ and a current density of $\sim 2$ $\mu a m p s / \mathrm{cm}^{2}$. The implantation energy for the Ag was chosen from calculations using TRIM 89[7] to target an energy that would have the same depth of implantation as the Sb.

Rutherford backscattering (RBS) measurements with $2.3 \mathrm{MeV} \mathrm{He}^{++}$ions were used to measure the depth profiles of implanted species. Transmission electron microscopy was done on all samples. Details of sample preparation are reported elsewhere.[8] Standard bright field (BF) 
imaging was used to observe the implanted layer and selected area electron diffraction was used to analyze the metal colloids formed in the silica wafers.

Linear optical absorption measurements were made at room temperature in air from 900 to $200 \mathrm{~nm}$ using a dual beam spectrometer (Cary 5). All samples were measured using an unimplanted sample in the reference beam. The absorption spectra were measured at three different positions on each sample and are reported as optical density (OD). The scatter in these measurements at these different positions was less than $1 \%$. The nonlinear index of refraction was measured at 596 and $617 \mathrm{~nm}$ for the samples using the Z-scan method.[9] The laser used for these experiments was a cavity dumped tunable dye laser with a 6 ps pulse duration. The laser was operated at $3.8 \mathrm{MHz}$. The average power was $250 \mathrm{~mW}$ for $596 \mathrm{~nm}$ and $200 \mathrm{~mW}$ for $617 \mathrm{~nm}$ in the $\mathrm{TEM}_{\infty}$ mode and the peak irradiance for a focal spot of $25 \mathrm{~mm}$ radius was $5 \times 10^{8} \mathrm{~W} / \mathrm{cm}^{2}$ for 596 $\mathrm{nm}$ and $4 \times 10^{8} \mathrm{~W} / \mathrm{cm}^{2}$ for $617 \mathrm{~nm}$. The error in these measurements was less than $20 \%$.

\section{RESULTS}

The distributions of ions as a function of depth from the surface are gaussian in shape with a peak at 0.14 microns for the Sb samples. The full width at half maximum (FWHM) for the Sb implanted sample is 0.14 microns. This value, 0.14 microns, was used to calculate values for $\mathrm{n}_{2}$ and $\alpha$. For the $\mathrm{Ag}$ implanted sample the distributions of ions as a function of depth from the surface is also gaussian in shape with a peak at 0.13 microns and FWHM of 0.09 microns.

Figure 1 and 2 show the bright field transmission electron micrographs for the $6 \times 10^{16} \mathrm{Sb}$ implanted sample and the $6 \times 10^{16} \mathrm{Ag}$ implanted sample respectively. Approximately spherical colloids are formed in both implantations.

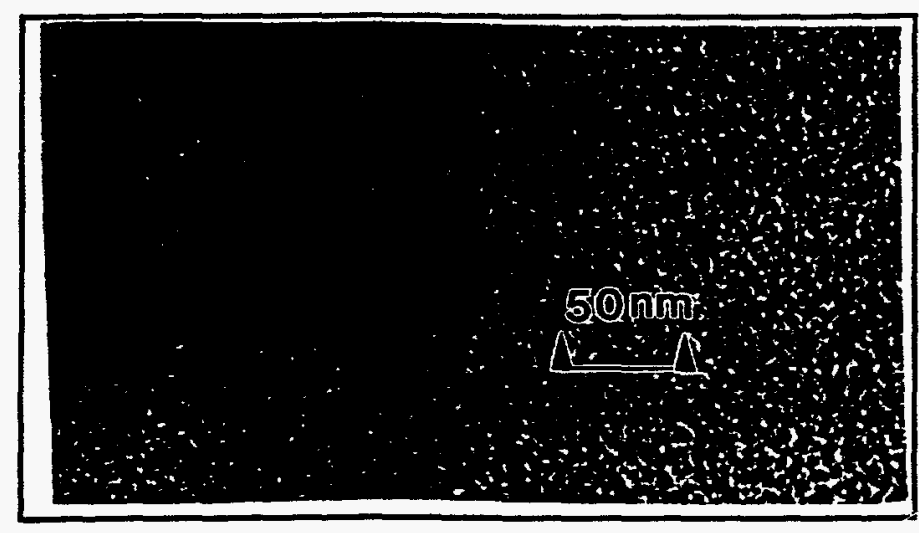

Figure 1: Electron transmission micrographs for sample implanted with $6 \times 10^{16}$ Sb sample. Mean particles size is $5.7 \pm 1.8 \mathrm{~nm}$ with a standard deviation of $1.6 \mathrm{~nm}$.

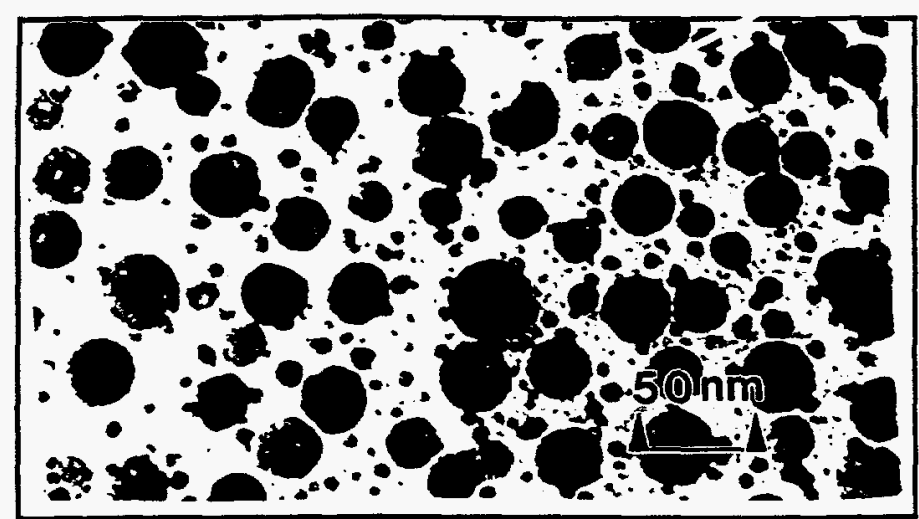

Figure 2: Electron transmission micrographs for sample implanted with $6 \times 10^{16} \mathrm{Ag}$ sample. Mean particles size is $13.5 \pm 1.8 \mathrm{~nm}$ with a standard deviation of $8.8 \mathrm{~nm}$. 
Figure 3 shows the optical spectra as a function of photon wavelength for the samples implanted with $\mathrm{Sb}$ and $\mathrm{Ag}$. The absorption in all the samples increases with decreasing wavelength with an approximate $1 / \lambda$ dependence and exhibits no distinguishing features. The total absorption throughout the spectral region increases with increasing dose. Figure 3 also compares the $6 \mathrm{Sb}$ and $6 \mathrm{Ag}$ implanted samples. The absorption spectra for the $\mathrm{Ag}$ implanted sample is dominated by an absorption peak at $\sim 400 \mathrm{~nm}$ and a shoulder at $\sim 520 \mathrm{~nm}$.

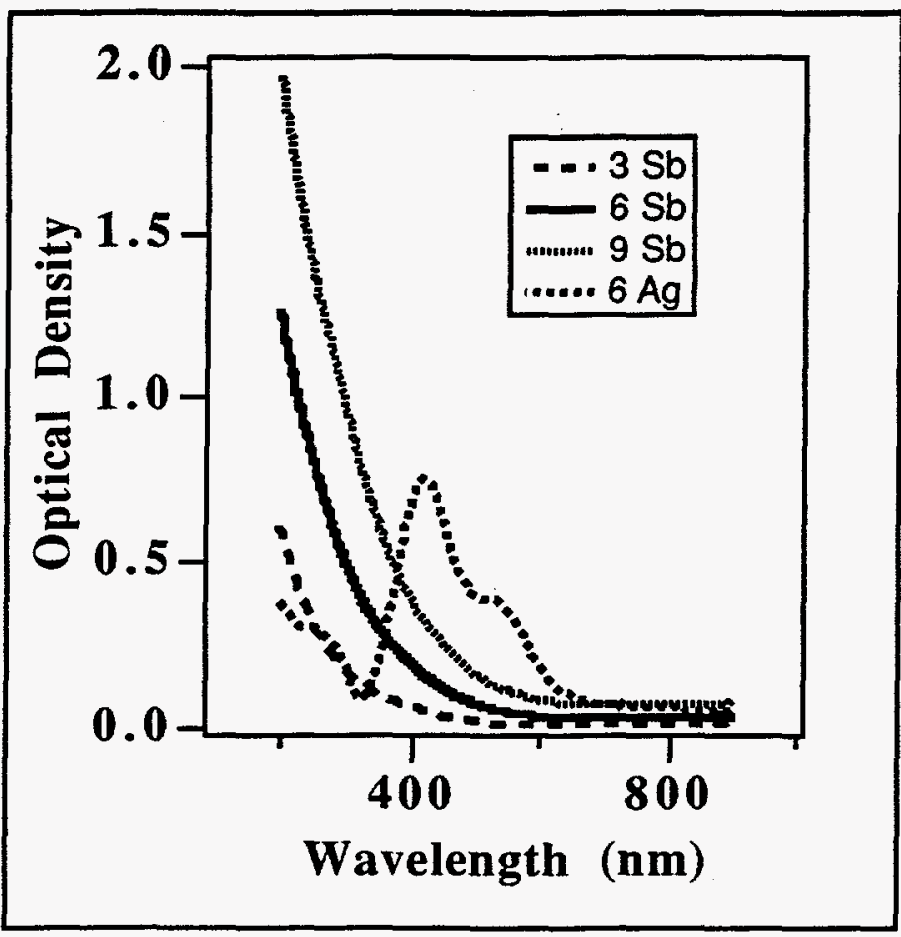

Figure 3: Optical density as a function of wavelength for samples implanted with 3,6 and $9 \times 10^{16} \mathrm{Sb}$ and a sample implanted with $6 \times 10^{16}$ Ag.

Table I lists the linear and nonlinear optical coefficients for the samples at $596 \mathrm{~nm}$ for all three Sb samples and the 6Ag sample. Table I also lists the linear and nonlinear optical coefficients for the samples at $617 \mathrm{~nm}$ for the $6 \mathrm{Sb}$ and $6 \mathrm{Ag}$ implanted samples. The nonlinear coefficients were calculated using the formalism from reference [8].

Table I

Linear and Nonlinear Optical Coefficients@ @ 596 nm and 617 nm

\begin{tabular}{|c|c|c|c|}
\hline $\begin{array}{c}\text { Sample } \\
10^{16} \text { ions } / \mathrm{cm}^{2}\end{array}$ & $\begin{array}{c}\alpha\left(\mathrm{cm}^{-1}\right) \times 10^{4} \\
(596 \mathrm{~nm} / 617 \mathrm{~nm})\end{array}$ & $\begin{array}{c}\mathrm{n}_{2}(\mathrm{~cm} 2 / \mathrm{W}) \times 10^{-9} \\
(596 \mathrm{~nm} / 617 \mathrm{~nm})\end{array}$ & $\begin{array}{c}\beta(\mathrm{cm} / \mathrm{W}) \times 10^{-4} \\
(596 \mathrm{~nm} / 617 \mathrm{~nm})\end{array}$ \\
\hline $3 \mathrm{Sb}$ & 0.13 & N.D. & N.D. \\
\hline $6 \mathrm{Sb}$ & $0.55 / 0.51$ & $0.4 / 0.4$ & N.D. / N.D. \\
\hline $9 \mathrm{Sb}$ & 1.36 & 0.9 & N.D. \\
\hline $6 \mathrm{Ag}$ & $3.17 / 2.20$ & $0.9 / 0.4$ & $-0.2 /$ N.D. \\
\hline
\end{tabular}


At $596 \mathrm{~nm}$ the linear absorption coefficient, $\alpha$, and the nonlinear index of refraction, $\mathrm{n}_{2}$, increase with increasing $\mathrm{Sb}$ concentration. In comparison, the $6 \mathrm{Ag}$ sample has the same $\mathrm{n}_{2}$ as the $9 \mathrm{Sb}$ sample however the $6 \mathrm{Ag}$ sample has more than twice the linear absorption. Nonlinear absorption was not detected in any of the Sb implanted samples, while the 6Ag sample shows a saturation of the nonlinear absorption, $\beta$, as indicated by the negative sign in Table I. At $617 \mathrm{~nm} \mathrm{n}_{2}$ for the $6 \mathrm{Ag}$ sample has decreased a factor of $\sim 2$ while its linear absorption decreased $\sim 30 \%$ compared to that at $596 \mathrm{~nm}$. The $\mathrm{n}_{2}$ for the $6 \mathrm{Sb}$ sample remains constant though $\alpha$ decreases by $\sim 10 \%$ from the values measured at $596 \mathrm{~nm}$.

\section{Discussion:}

The optical properties of these metal colloid -glass composites can be described using effective medium theory for small non interacting particles in a dielectric. [1,2] The linear response for colloids with diameters less than $\lambda / 20$, where $\lambda$ is the wavelength of the incident radiation, is reasonably described by effective medium theory in the electric dipole approximation $[1,10]$ and is given by

$$
\alpha=\frac{18 \pi n_{d}^{3}}{\lambda} \cdot \frac{p \varepsilon_{2}}{\left[\varepsilon_{1}+2 n_{d}^{2}\right]^{2}+\varepsilon_{2}^{2}}
$$

where $\alpha$ is the absorption coefficient, $\varepsilon(\lambda)=\varepsilon_{1}+i \varepsilon_{2}$ is the dielectric constant of the metal, $p$ is the volume fraction of the metal particles and $n_{d}$ is the index of refraction of the dielectric host. The absorption is expected to exhibit a maximum at the surface plasmon resonance frequency for which the condition $\varepsilon_{1}+2 n_{d}^{2}=0$ is met. The surface plasmon resonance frequency depends on the electronic properties of the metal colloids and on the index of refraction of the host dielectric, $\mathrm{n}_{\mathrm{d}}$. The third order nonlinear susceptibility, $\chi_{\text {eff }}^{(3)}$, can be expressed as[1]

$$
\chi_{\text {eff }}^{(3)}=p f_{c}^{2}(\omega)\left|f_{2}(\omega)\right|^{2} \chi_{m}^{(3)} \quad \text { with } \mathbf{f}_{c}(\omega)=\frac{3 n_{d}^{2}}{\varepsilon_{1}+2 n_{d}^{2}}
$$

where $f_{c}(\omega)$ is the local field factor and $\chi_{m}^{(3)}$ is the nonlinear susceptibility of the metal colloids. The index of refraction and the intensity dependent term are related to the above quantities by[11]

$$
\mathrm{n}=\mathrm{n}_{\mathrm{o}}+\mathrm{n}_{2} \mathrm{I} \quad \text { and } \quad \mathrm{n}_{2}=\frac{12 \pi}{\mathrm{n}_{\mathrm{o}}} \operatorname{Re}\left[\chi_{\mathrm{eff}}^{(3)}\right]
$$

where $\mathrm{n}_{\mathrm{o}}$ is the linear index of refraction and $\mathrm{n}_{2}$ is the intensity dependent component. From equation (2) a potentially large enhancement of the effective nonlinear susceptibility and in turn $n_{2}$ near or at the surface plasmon resonance frequency is possible due to local field effects.

While part of the linear absorption in the ultraviolet may be attributed to defect centers, based on prior work we expect that the absorption will be dominated by the presence of the metal colloids.[10]

From equation (1) the optical properties depend explicitly on the electronic properties of the metal colloids through $\varepsilon(\lambda)$ and on the index of refraction of the host dielectric, $n_{d}$, while depending implicitly on particle size through $\varepsilon(\lambda)$. Assuming that $n_{d}$ remains constant for the host 
dielectric, we will not consider it further and will instead consider the metal particle contributions to the absorption.

From Figure 1 the TEM measurements reveal that the particles are approximately spherical in shape for all samples. Because the shape is similar for all implants we do not expect any changes in the optical spectra based on particle shape differences. However the colloids are larger for the 6Ag implanted sample than for the Sb implanted samples.

For $\mathrm{Sb}$ implanted samples there is no indication of a surface plasmon resonance absorption in the optical absorption spectra. We attribute the increasing absorption with decreasing photon wavelength in these samples to the formation of Sb nanometer dimension colloids. The dielectric properties of the $S b$ clusters are such that the condition $\varepsilon_{1}+2 n_{d}^{2}=0$ is not satisfied[5] and therefore these samples do not exhibit a distinct surface plasmon resonance absorption feature. However the spectra does exhibit an approximate $1 / \lambda$ dependence behavior expected from equation (1) for nanometer dimension colloids. We attribute the increasing absorption with dose to the increase in volume fraction of metal colloids formed with increasing dose.

The optical spectra of the $6 \mathrm{Ag}$ sample exhibits the characteristic absorption due to the surface plasmon resonance of nanometer dimension Ag colloids.[7,10,12] We attribute the peak at $400 \mathrm{~nm}$ in the $6 \mathrm{Ag}$ sample to Ag colloids $<25 \mathrm{~nm}$ in diameter.[10,12] With an increase in particle size additional higher order terms than the dipole term from Mie theory are required to describe the absorption observed. We attribute the shoulder at $520 \mathrm{~nm}$ in the $6 \mathrm{Ag}$ sample to the quadrupole term from Mie theory.[7,10,12]

The optical properties of the $\mathrm{Sb}$ implanted samples are clearly different than those of the $\mathrm{Ag}$ sample. From equation (1) the volume fraction of the metal present and the dielectric function, $\varepsilon(\lambda)=\varepsilon_{1}+i \varepsilon_{2}$, of the metal clusters are expected to effect the absorption. We readily observe the effect of the volume fraction of metal colloids by the increase in absorption with dose in the $\mathrm{Sb}$ implanted samples. Colloid size and volume fraction increase with dose. For increasing dose more ions are found in colloid form. While some of the ions may be in colloids too small to be observed ( $<2 \mathrm{~nm}$ in diameter) in the lower doses the increase in dose reduces the presence of these smaller colloids.[8] This reduction is thought to occur from the additional heating with the longer implantation time of the larger doses. The total concentration of metal ions in the $6 \mathrm{Sb}$ and $6 \mathrm{Ag}$ samples is the same. The difference in the optical properties are then the result of a different electronic structure.

The nonlinear index of refraction, $\mathrm{n}_{2}$, at $596 \mathrm{~nm}$ increases with the dose in the $\mathrm{Sb}$ implanted samples. Both the linear absorption and $n_{2}$ increase approximately at the same rate with dose. These changes are in reasonable agreement with equation 1 and 2 that show that $\alpha$ and $\mathrm{n}_{2}$ increase with volume fraction of the metal ions. Nonlinear absorption is not detected in the Sb implanted samples. The $\mathrm{n}_{2}$ for the Ag implanted sample has the same value as for the $9 \mathrm{Sb}$ implanted sample but with larger linear absorption and a saturated nonlinear absorption.

The value for $\mathrm{n}_{2}$ at $617 \mathrm{~nm}$ has not changed for the $6 \mathrm{Sb}$ compared to that at $596 \mathrm{~nm}$. The value for $\mathrm{n}_{2}$ at $617 \mathrm{~nm}$ for the $6 \mathrm{Ag}$ sample decreases $60 \%$. While the $\mathrm{n}_{2}$ 's for the $6 \mathrm{Sb}$ and $6 \mathrm{Ag}$ samples are the same at $617 \mathrm{~nm}$ the $6 \mathrm{Ag}$ sample has $\sim 4$ times the linear absorption. The lack of change in $\mathrm{n}_{2}$ for the $6 \mathrm{Sb}$ sample and the rapid decrease in $\mathrm{n}_{2}$ for the $6 \mathrm{Ag}$ with wavelength suggest that different mechanisms may dominate the nonlinear responses for these elemental colloids.

Reference 1 gives a review of the various mechanisms. Using $\mathrm{n}_{2} / \alpha$ as a figure of merit (FOM), at $596 \mathrm{~nm}$ the $6 \mathrm{Sb}, 9 \mathrm{Sb}$ and $6 \mathrm{Ag}$ samples have FOM of $0.7,0.6$ and 0.3. As the Sb samples do not have a surface plasmon resonance and we are far off the surface plasmon resonance frequency for the Ag colloids we anticipate only minimal enhancements of the nonlinear response due to local field effects. We conclude the behavior of the values of $\mathrm{n}_{2}$ as a function of $\lambda$ arise from differences in the $\chi_{\mathrm{m}}^{(3)}$ of the metal colloids. These results suggest there are metal nanometer dimension colloids, that while not showing a SPR enhancement at wavelengths away from the SPR, can have better figures of merit.

The z-scan technique measures both the thermal and electronic contributions to the nonlinear index. Previous nonlinear measurements on $\mathrm{Cu}$ colloids with similar absorption formed by ion implantation have been reported.[8] Using similar wavelength, power, repetition rate and pulse width of the laser the thermal loading of the sample was found not to dominant the response 
because of the relatively iong interpulse spacing ( $263 \mathrm{~ns}$ ) even though the $6 \mathrm{ps}$ pulse duration is comparable to the electron thermalization times of the $\mathrm{Cu}$ colloids.[8] Based on the above discussion we conclude that the nonlinear response is either dominated by or has a significant contribution from electronic mechanisms.

\section{CONCLUSIONS}

From these results we suggest that some metallic colloids even though they do not exhibit a surface plasmon resonance frequency may be better for devices because of lower linear absorption yielding substantially better figures of merit for device applications.

\section{ACKNOWLEDGMENTS}

The authors wish to thank Prof. R.F. Haglund for the use of his laboratory for the nonlinear measurements. The authors acknowledge the support of the Army Research Office under grant DAAH04-93-G-0123 and Oak Ridge National Laboratory, managed by Lockheed Martin Energy Research Corp. for the U.S. Department of Energy under contract number DE-AC0596OR22464.

\section{REFERENCES}

1. C. Flytzanis, F. Hache, M.C. Klein, D. Ricard and Ph. Roussignol, Progress in Optics, 29 (1991) 321 and references there in.

2. R.H. Magruder,III, R.F. Haglund, L. Yang, C.W. White, Lina Yang, R. Dorsinville and R.R. Alfano. J.Appl. Phys. Lett. 62 (1993) 465.

3. V. Mizrahi, K.W. DeLong, G.I. Stegeman, M.A. Salfi and M.J. Andrejcu, Opt. Lett., 14 (1989) 1140.

4. G.I. Stegeman and R.H. Stolen, J. Opt. Soc. Am. B6 (1989) 652.

5. J. Alan Creighton and D.G. Eadon, Chem. Soc. Faraday Trans., 87 (1991) 3881.

6. Z. Pan, S.H. Morgan, D.O. Henderson, S. Park, R.A. Weeks, R.H. Magruder,III and R. A. Zuhr, Journal of Optical Materials, 4 (1995) 675. .

7. J.P. Biersack and L.G. Haggmark, Nucl. Inst. Methods B 174 (1980) 257.

8. R.H. Magruder,III, R. F. Haglund, L.Yang,J.E. Wittig and R.A. Zuhr, J. Appl. Phys., 76 (1994) 715.

9. M. Sheik-Bahae, A. A. Said and E. W. VanStryland, Opt. Lett. 14 (1989) 955.

10. C.F. Bohren and D.R. Huffman, Absorption and Scattering of Light by Small Particles, John Wiley and Sons, New York (1983) .

11. M.J. Weber, D. Milam and W.L. Smith, Optical Eng., 17 (1978) 463.

12. M. Vollmer and U. Kreibig, Nuclear Physics Concepts in the Study of Atomic Cluster Physics, R. Schmidt, H.O. Lutz and R. Dreizler, eds., Springer - Verlag, Berlin , 1992. 